\title{
Longitudinal Study of Pandemic and Natural Disaster Distress
}

\author{
Joseph Bulbulia $^{\mathrm{a}, \mathrm{b}, \mathrm{f}, 1}$, Sofia D. Piven ${ }^{\mathrm{b}}$, Lara M. Greaves ${ }^{\mathrm{b}}$, Danny Osborne ${ }^{\mathrm{b}}$, Geoffrey Troughton ${ }^{\mathrm{a}}$, Kumar Yogeeswaran $^{\mathrm{d}}$, and \\ Chris G. Sibley
}

${ }^{a}$ Victoria University of Wellington, Wellington 6140 , New Zealand; ${ }^{b}$ University of Auckland, Auckland 1142, New Zealand; ${ }^{\mathrm{d}}$ University of Canterbury, Christchurch 8140 ,

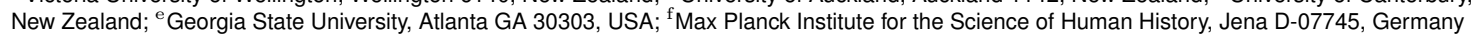

This manuscript was compiled on November 17, 2020

TBA

Alert Level-4|Anxiety | Bayesian | Employment| Kessler-6 | Longitudinal |Stan |Well being

N ew Zealand's Alert Level-4 lockdown extended from midnight March $25^{\text {th }}$ to midnight April $27^{\text {th }}, 2020$, effectively quarantining the entire country. An independent global stringency evaluation ranked the country's lockdown as the world's $8^{\text {th }}$ most restrictive, marking the lockdown 96.30 severity points out of a possible 100 points (1). Such restrictions were of unknown duration and efficacy. The country's fragile tourism and export-economies were immediately imperilled. Only essential workers could work. COVID-19 cases increasing geometrically after lockdown started, with a geometric grow in COVID-19 deaths (S1.A). Thus, New Zealand's early COVID-19 lockdown simultaneously combined (1) mandated physical-distancing with (2) mortal health risks and (3) widespread loss of employment. Any such condition could provoke psychological distress.

Not surprisingly, research in New Zealand, Australia, China, and the United States finds that COVID-19 increased psychological distress as measured by the Kessler-6 inventory(2-7). However, psychological distress among New Zealanders was lower and less reliable than it was elsewhere. It has been theorised that the lethal health risks posed by SARS-CoV-2 virus produced distress $(5,7)$. It has been speculated that loss of employment and economic downturn elevated distress (7). However, at the time of New Zealand's early Alert Level-4 lockdown, health risks and economic uncertainties were comparable to those in other countries (S1.A). It has been speculated that confidence in the government(6), a sense of neighbourhood community(6) and strong social belonging (8) might have mitigated psychological distress. Additionally, it is plausible that robust economic and health protections during stringent pandemic lockdown may afford relief even among those who are low in these indicators. Presently, however, theories pandemic distress mitigation remain untested.

Here, we compare longitudinal responses from the New Zealand Attitudes and Values Study (NZAVS), March $26^{\text {th }}-$ April $12^{\text {th }}, 2020$ (lockdown) with participants' pre-COVID-19 baselines from the previous year ( $N=940$, sample details: (S1.B). This random, nationally diverse sample obtained as part of routine data collection for the NZAVS affords a powerful tool for investigating pandemic distress mechanisms for the following reasons: (1) New Zealand's lockdown required sheltering-in-place, isolating most residents from those living outside their homes; (2) lockdown occurred in a setting of economic uncertainty; (3) during the early period of lockdown from which this sample was randomly drawn, health risks were escalating, as both infectious case numbers and COVID-19 deaths began to climb geometrically (S1.A); (4) there is variation in longitudinal indicators of public confidence in the government, business satisfaction, a sense of neighbourhood connection, health satisfaction, and social belonging, with established pre-COVID baselines in our random sampling design, enabling robust inference about uncertainties in theoretically postulated mechanisms of psychological burden and relief (see S1.B,S4); (5) finally, the availability of relevant indicators from previous NZAVS waves enables us to quantify mental health trajectories following the 2011 Christchurch earthquakes, and thus to better identify unique features of pandemic distress production and mitigation (year: 2011, $N=6,806$ )(see: Methods) 

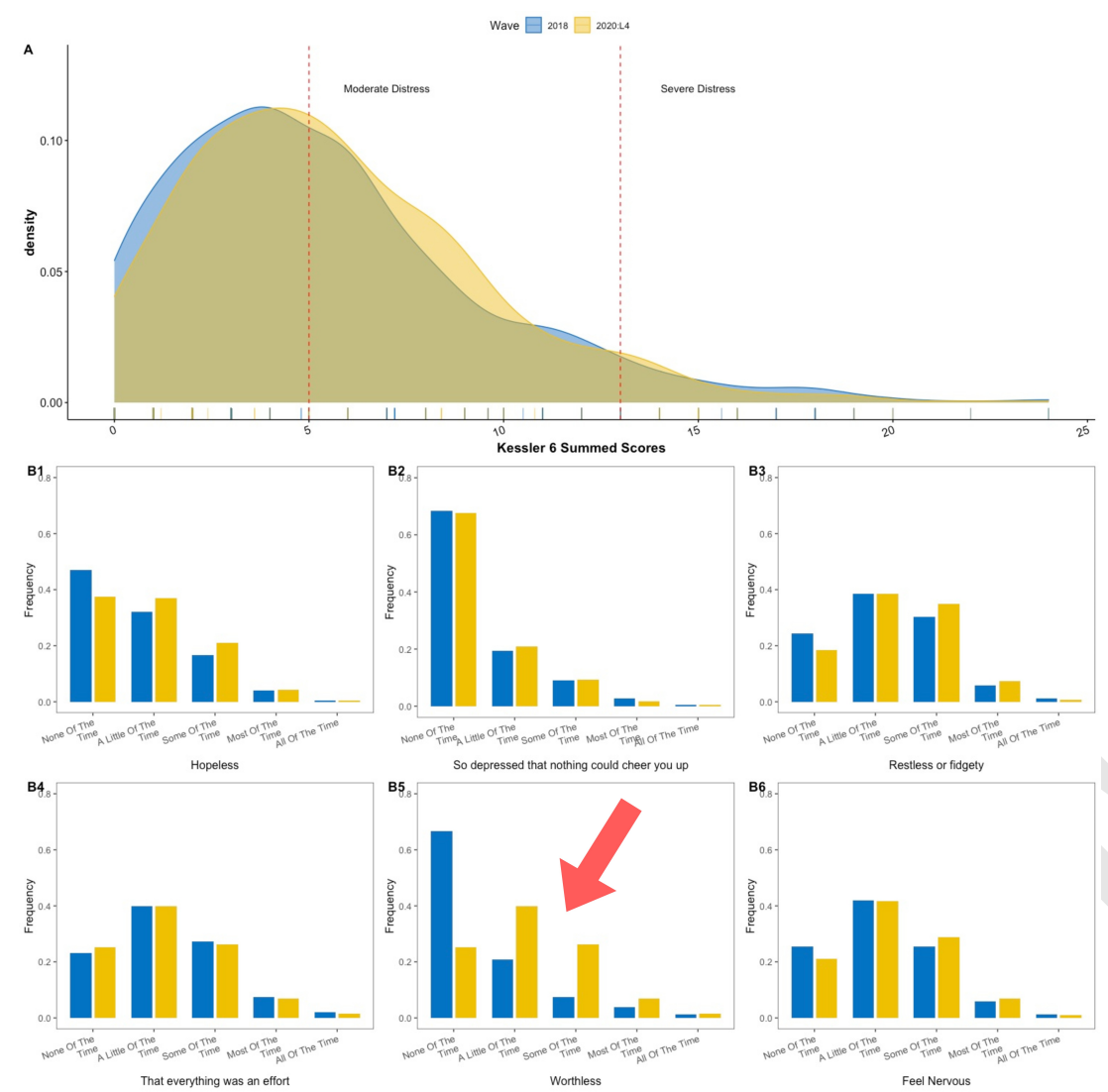

Fig. 1. Exploratory sample analysis of COVID-19 lockdown distress reveals (A) increases in the density of summed Kessler-6 distress scores with movement to the moderate distress range (blue $=$ pre-pandemic baseline; gold = COVID-19 lockdown). Panels B1-B6: Histogram of responses changes within participants comparing 2018 baselines (blue) and COVID-19 lockdown responses (gold). Large increases in feelings of worthlessness are evident (B5).

\section{Results}

Exploratory analysis reveals increases in the density of summed Kessler-6 psychological distress scores during COVID-19 lockdown, with a movement toward the moderate distress range, see Figure1A. Consistent with previous research we observe an increase in overall summed Kessler-6 psychological distress during COVID-19 lockdown [HDI: 0.01, 0.09; pd: 98.1\%] (S2 Model 1). Additionally, during the lockdown (yellow), feelings of worthlessness markedly increased (blue), see Figure 1B5. These findings contrast with previous research in the United States, which find variable effects of lockdown across most Kessler-6 items, with specific challenges to feelings of hopelessness, sadness, as well as worthlessness(7).

Bayesian Multilevel Ordinal Regression. To systematically quantify psychological distress trajectories during COVID-19 lockdown across the six indicators of the Kessler-6 psychological distress scale (S1), we apply multilevel multivariate Bayesian ordinal regression models to nationally diverse pre-COVID-19/ and COVID-19 lockdown panel responses. We regress all six individual Kessler-6 distress indicators against the five theoretically postulated distress-mitigation indicators within the same model. We use the interactions of each distress-mitigation indicator with the measurement-interval indicator (pre/during lockdown) to estimate functional differences in distress-mitigator performance. We infer parameter uncertainty using a Bayesian approach, including weakly informative priors and posterior model checks $(9,10)$ (see Method, S2). Figure 2A presents results.

Depression. We do not observe a main effect of stringent-lockdown for feelings of depression [HDI: -0.26, 0.20, pd 56.8\%]. However, we observe a reliable interaction of stringent-lockdown and sense of neighbourhood community for feelings of depression [HDI: 0.16, 0.61, pd: 99.7\%]. As indicated in Figure 2C5, in 2018, those with a lower sense of community were less likely to respond that they were depressed "none of the time" and more likely to respond they were depressed "a little of the time." During lockdown, this predictive 
relationship flattened, with those at the lower end the sense of community-scale more likely to respond that they were depressed "none of the time." In other words, during the lockdown, a sense of community was no longer predictive of feelings of depression. Quite the opposite, during the lockdown, there was relief among those low in a sense of neighbourhood community.

Nervousness. There was a main effect of stringent-lockdown for feelings of nervousness, which is less reliable and smaller in magnitude than changes in other Kessler-6 items [HDI: 0.01, 0.36, pd: 95.0\%]. We do not find evidence for an interaction between stringent-lockdown and any of the other mitigation-indicators for nervousness.

Hopelessness. We observe a main effect of stringent-lockdown for greater feelings of hopelessness [HDI: 0.35, 0.72 , pd: $100.0 \%]$.

There was an interaction between stringent-lockdown and social-belonging for hopelessness [HDI: 0.06, 0.47, pd: 98.1\%]. As indicated in Figure 2B1, the buffering effects of high social belonging for hopelessness were attenuated during the lockdown, with such attenuation more pronounced at the high end of social belonging. Those who were low in social belonging remained constant in their hopelessness during the lockdown.

Additionally, there was an interaction between stringent-lockdown and business satisfaction for hopelessness [HDI: 0.02, 0.44, pd: 95.4\%]. As indicated in Figure 2B2, people at the low end of business satisfaction were relatively lower in felt hopelessness during lockdown compared with their 2018 baselines and people at the high end of business satisfaction experienced greater hopelessness during lockdown compared with their 2018 baselines. Even high business satisfaction was unable to defeat occasional feelings of hopelessness.

Restlessness. We observe a main effect of stringent-lockdown for increasing feelings of restlessness [HDI: 0.29, 0.64, pd: 100.0\%].

Additionally, there was an interaction between stringent-lockdown and business satisfaction for feelings of restlessness [HDI: 0.01, 0.40, pd: 94.6\%], see Figure 2D2. Whereas in 2018 people who were high in business satisfaction were more likely to feel restless "none of the time" and less likely to feehttps://www.overleaf.com/project/5f03aa5b47bd100001db28291 restless "some of the time," this predictive relationship weakened during the lockdown. Despite high business satisfaction, small increases in hopelessness were evident.

Everything is an Effort. A silver lining for mental health is evident from the main effect of stringent-lockdown for diminishing feelings that everything is an effort [HDI: -0.44, -0.10, pd: 99.3\%]. Moreover, stringentlockdown altered the performance of every distress mitigator except satisfaction with the government.

There was an interaction between stringent-lockdown and social-belonging for feelings of effort [HDI: -0.50, -0.13, pd: 99.7\%], see Figure 2E1. Compared with 2018, higher social belonging predicted fewer feelings that everything was an effort, whereas lower social belonging predicted greater feelings that everything was an effort. Thus, while lockdown brought relief through reduced experiences that everything was an effort, greater social belonging was a pathway to greater relief.

There was an interaction between stringent-lockdown and business satisfaction for feelings of effort [HDI: 0.04, 0.42, pd: 97.3\%], see Figure 2E2. Whereas in 2018, lower business satisfaction was associated with greater feelings of effort "some of the time", this tendency diminished, again with more relief experienced at the low end of business satisfaction.

There was an interaction between stringent-lockdown and health-satisfaction for feelings of effort [HDI: 0.11, 0.47, pd: 99.7\%], see Figure 2E4. Compared with 2018 baselines, those at the low end of health satisfaction during lockdown were less likely to report feeling effort "most of the time" and more likely to report feelings of effort "a little of the time." Thus despite low heath-satisfaction, people during lockdown experience fewer experiences of effort.

There was an interaction between stringent-lockdown and a sense of neighbourhood community for feelings of effort [HDI: 0.07, 0.42, pd: 98.7\%], see Figure 2E5. Notably, lockdown brought greater relief 
from feelings of effort among those lower in their sense of community.

Worthlessness. We observe a remarkably strong main effect of stringent-lockdown for increasing feelings of worthlessness [HDI: 2.04, 2.50, pd: 100.0\%].

There was an interaction between stringent-lockdown and social belonging [HDI: 0.31, 0.72, pd: 100.0\%], see Figure 2F1. Specifically, there was a strong attenuation between high social belonging and perceived feelings of worthlessness "none of the time" and "a little of the time" and a strong elevation in perceived feelings of worthlessness "some of the time" and "most of the time" among those low in social belonging. Put differently, low social belonging is a risk factor for even greater experiences of worthlessness.

There was an interaction between stringent-lockdown and health satisfaction for feelings of worthlessness [HDI: $0.05,0.42$, pd: $97.4 \%$ ], see Figure 2F4. We observe an attenuation in the relationships between high satisfaction and perceived feelings of worthlessness "none of the time" and "a little of the time" as well as a strong elevation perceived feelings of worthlessness among those low in health satisfaction.

There was an interaction between stringent-lockdown and neighbourhood community for feelings of worthlessness [HDI: 0.02, 0.39, pd: 95.8\%], see Figure 2F5. Even a high sense of neighbourhood community no longer reduced feelings of worthlessness as they did in 2018.

\section{Discussion}

We observe, first, that although New Zealand's stringent early COVID-19 lockdown occurred in a setting of national economic and health uncertainties, residents experienced only modest increases in feelings of hopelessness, restlessness, and nervousness. Moreover, there was no increase in severe depression. By contrast, cohort studies from the United States during the early 2020 COVID-19 pandemic reveal elevated mental distress in the United States' adult population(7). Indeed, compared with responses from the previous year's cohort, feelings of hopelessness, sadness, and worthlessness were over four times more likely during the early U.S. pandemic. Additionally, feelings of nervousness, restlessness, and effort increased $(4,7)$. Twenge and Joiner (7) speculate that employment obstacles and home-isolation were potential contributing factors to such early COVID-19 distress across the U.S. However, New Zealand's shelter-in-place pandemic lockdown resulted in more pronounced isolation for a longer period of time. During New Zealand's Alert Level-4 all but essential employment outside the home ceased. The causes of elevated distress in the United States are not employment-obstacles and home isolation. Moreover, in New Zealand, even those low in business satisfaction, low in health satisfaction, low in a sense of community, and low in government satisfaction experienced distress buffering. We infer that the augmented psychological distress reported in the United States arose from insufficient income protections and insufficiently protective physical distancing mandates in the United States. The difference-maker for mental health protection in New Zealand appears to have been the government's combination of robust economic support with a severely restrictive infectious disease intervention that immediately addressed the public health hazard. Importantly, New Zealand residents did not need to be satisfied with the government for their mental health to benefit from the country's supportive and stringent public health response.

Second, during New Zealand's lockdown, feelings that everything was an effort declined. However, not everyone benefited equally. To experience relief from feelings of effort, a sense of social-belonging was crucial. Indeed, those low in social-belonging experienced more considerable effort (Figure 3A2). Intriguingly, those low on health-satisfaction experienced relief from feelings of effort (Figure 3B2). On reflection, it is not surprising that a government-imposed shelter-in-place mandate committed to eradicating lethal infectious disease would bring relief to those low in health satisfaction. Sheltering-in-place reduced physical effort and removed those susceptible to a deadly infectious disease from harm's way.

Third, although New Zealander's suffered less than residents of other countries, they did not dodge every mental health bullet. During New Zealand's lockdown, feelings of worthlessness increased threefold on the year's previous ratings. Before the COVID-19 pandemic, New Zealand's employment levels were high. However, during lockdown, all but essential workers were either disrupted from their ordinary employment 
routines or unemployed. Such employment disruption was of indefinite duration. Previous mental health research finds that unemployment diminishes mental health (11). For example, among those who are unemployed, clinical levels of psychological distress are twice as high (12). A long history of unemployment research reveals that a loss of routine is among the causes of poor mental health among those without work $(13,14)$. We predict that the impact of heightened feelings of worthlessness among New Zealanders in the aftermath of COVID-19 will turn on the persistence and magnitude of New Zealand's unemployment.

Fourth, contrary to our previous predictions (6), we do not find distress mitigation from satisfaction with the government or a strong sense of community. Moreover, satisfaction with business was only weakly predictive of positive mental health, with small relative gains experienced by those low in business satisfaction. During New Zealand's lockdown, whether a New Zealander had confidence in their government, was satisfied with business, or felt connected with their neighbourhood community was not informative of their mental distress levels. We infer that the mitigation of pandemic lockdown-distress does not require changing a population's attitudes to government, business, and community.

Fifth, although high levels of social belonging could not prevent feelings of worthlessness during the lockdown, social-belonging was critical to mitigating psychological distress across all Kessler-6 indicators. Moreover, social belonging functioned as a pathway for relief from feelings that everything is an effort. Indeed, those who felt lower social belonging during lockdown manifested greater feelings of effort compared with pre-COVID-19 baselines. Thus, although general feelings of greater confidence in government and sense of neighbourhood community were not predictive of mental health mitigation during lockdown, social belonging with people one already knows protected people from an greater elevation in psychological distress. This finding supports Van Bavel et al.'s (2020) theory that social belonging is a crucial to mental health during a pandemic lockdown (8). We infer that to optimise mental health during a pandemic requires combining the health assurances of restrictive physical-distancing while also preserving experiences of social belonging. To improve mental health during pandemics, applied mental health researchers should develop strategies for balancing this challenge.

To conclude, the generally diminished mental health challenge observed in New Zealand compared with that of other countries demonstrates that even a highly restrictive pandemic lockdown need not cause severe psychological distress. Moreover, that distress mitigation in New Zealand was apparent even among those who were low in government confidence, low in business satisfaction, and low in a sense of neighbourhood community reveals that pandemic distress mitigation does require changing a population's political attitudes, business outlooks, or civic sensibilities. Rather, pandemic distress mitigation arises from delivery of basic income and health protections. One reason a stringent lockdown protects mental health is that it reduces the psychological distress of lethal infectious disease. However, an effective lockdown requires isolating people. Our systematic longitudinal test of theorised pandemic distress-mitigation mechanisms supports previous speculation that perceived interpersonal belonging is crucial for preserving psychological health (8). Put simply, we show that optimising mental health during a pandemic requires restrictive physical distancing that minimises social-psychological distancing. Developing effective strategies for addressing the cognitive and motivational challenges of this dual imperative is an important new horizon at the boundary of the behavioral sciences, engineering, and public health.

\section{Materials and Methods}

Sample. The NZAVS is a national longitudinal panel study that has been tracking social attitudes, personality, and health outcomes within individuals since $2009(N s=4,441-47,951)$. Here, we conduct a novel analysis of the rolling 2020 data-frame sample composed of 940 longitudinal participants who responded to both Wave 10 (2018) and Wave 11 (2020) of the NZAVS, which were taken respectively before and during the early stages of the lockdown in New Zealand. This dataset was first used to measure changes in institutional trust and wellbeing during lockdown (6) (Methods). The Time 10 (2018) Wave of the NZAVS contained responses from 47,951 participants (18,010 retained from one or more previous waves). By systematically investigating demographic and mental health differences between this sample and the larger 2018 sample, we investigate and find no evidence for potential confounding biases in the 
online lockdown sample (S4).

Psychological Distress Buffers. We investigate satisfaction with business and satisfaction with one's own health to assess the role of economic and health uncertainty in moderating distress. We investigate satisfaction with the government and a sense of neighbourhood community to evaluate hypotheses that these parameters insulated people from psychological distress (6). We investigate the buffering effects of social belonging because previous research reveals strong correlations with mental health (15), and because social belonging is specifically theorised to mitigate distress from pandemic physical distancing strategies (8). We use the Kessler-6 psychological distress scale to quantify dimensions of mental health challenges because it is a clinically validated tool for screening anxiety and depression with good diagnostic concordance across cross-cultural samples $(16,17)$ (More about our measures and descriptive statistics in S1).

Multi-level Ordinal Model. We follow Bürkner and Vuorre (18) and Liddell and Kruschke (19) in advising the use of ordinal models for non-metric data such as the Kessler-6 items. Here we employ a categorical model in which the observed variable y has a probability of being equal to response option +1 latent thresholds. An ordinal model assumes that an observed ordinal indicator measures a latent continuous variable (18, 20). The Kessler- 6 has five ordinal response options for indicating frequency of feeling distress during the past 30 days: "None Of The Time", "A Little Of The Time", "Some Of The Time", "Most Of The Time", "All Of The Time" hence six thresholds. The Kessler-6 distress indicators are: "Feeling Hopelessness;" "Feeling so Depressed Nothing Could Cheer You Up;" "Feeling Nervous or Fidgety;" "Feeling Everything is an Effort;" "Feeling Worthless;" "Feeling Nervous." We simultaneously modelled each of these six outcomes within a single model. Below, the superscript $k$ is denotes the $k=1 \ldots 6$ Kessler- 6 outcomes. Residuals for cumulative categorical models are not identified, so are fixed to $1 . \alpha_{j 0}^{k}$ denotes the $t=1 \ldots 5$ intercepts estimated for the ordinal model, which assumed a cumulative logit distribution for the outcome, and where the lowest response level is modelled as zero, hence four intercepts are estimated for each Kessler- 6 response indicator. $\alpha_{c}^{k}$ denotes the grouping intercept for the repeated measures within individuals.

\section{$\beta$}

denotes the individual-level predictors. The linear predictors are given in equation $\mathrm{S} 1$, where 'C' identifies an indicator is centered and 'S' identifies an indicator is centered and scaled. The statistical model and priors are given in equation 2

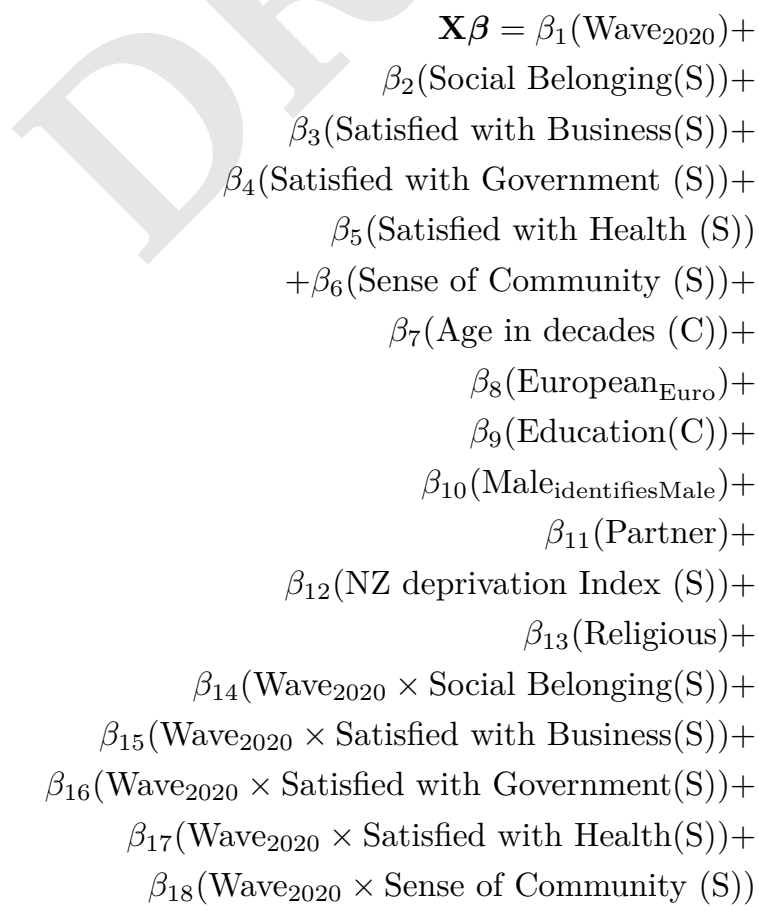




$$
\begin{array}{r}
y^{k} \sim \operatorname{Ordered}\left(\mu_{t}^{k}\right) \\
\operatorname{CumLogit}\left(\mu_{t}^{k}\right)=\alpha_{t}^{k}+\beta^{k} \\
\alpha_{t}^{k}=\alpha_{t 0}^{k}+\alpha_{c}^{k} \\
\alpha_{t 0}^{k} \sim \operatorname{StudentT}_{(3,0,10)} \\
\alpha_{c}^{k} \sim \operatorname{StudentT}^{+}(3,0,10) \\
\boldsymbol{\beta}^{k} \sim \operatorname{Normal}(0,1)
\end{array}
$$

Posterior predictive checks reveal that the categorical logistic distribution is a good choice.4

Comparative Analysis of Natural Disaster Distress Shows Uniqueness of Lockdown Distress. In 2010 and 2011, the New Zealand Canterbury region was struck by two major earthquakes, the latter of which would claim its place as one of New Zealand's most lethal and damaging natural disasters. On September 4 of 2010, a magnitude 7.1 earthquake hit Christchurch. Urban areas suffered major structural damages and liquefaction, though without human casualties. Efforts to rebuild were made difficult by a long series of aftershocks. During reconstruction in the summer of 2011, a 6.3 magnitude aftershock hit near the central business district on the afternoon of February 22, claiming 185 lives and causing over NZD \$11B in structural damages. Office buildings, historic churches, and buildings affected by the previous earthquake collapsed or were damaged beyond repair. The challenge of rebuilding New Zealand's second-largest city was estimated at over NZD \$20B (21). The distress of the earthquakes caused a regional religious revival (22).

To clarify the unique effects of the COVID-19 lockdown for psychological distress, we applied a parallel investigation of distress following this natural disaster New Zealand's Christchurch earthquakes $\left(\mathrm{n}_{\text {notaffected }}=5372, \mathrm{n}_{\text {affected }}\right.$ $=1434)$. To assess individuals affected by the Christchurch earthquakes, the NZAVS Time 3 (2011) survey asked: "Were you personally affected by the Christchurch earthquakes?" (yes or no). We coded "yes" as "1" and "no" as "0." 1434 participants $(N=6806)$ reported being personally affected by the Christchurch earthquakes (Supplement 2$)$.

Exploratory analysis suggests that elevated psychological distress among earthquake-affected people occurred in the moderate and high distress registers, implying that the mental health challenge from this natural disaster may have exceeded that of COVID-19 lockdown, see Figure 5A/B. As evident in Figure 5B panels 1-6, exploration of responses across the Kessler- 6 items reveals consistent signatures of low elevation across all dimensions. This contrasts with the attack evident during COVID-19 lockdown on feelings of worthlessness (Figure 5A5).

Next, we quantified distress signatures across the six Kessler-6 items among those affected/not affected by the Christchurch earthquakes, see Figure 6. We observe main effects of earthquake exposure on psychological distress across each Kessler-6 dimension: feelings of hopelessness [HDI: 0.08, 0.28, pd: 99.8\%], depression [HDI: 0.08, 0.32, pd: 99.7\%], restlessness [HDI: 0.09, 0.27, pd: 99.9\%], that everything was an effort [HDI: 0.03, 0.21, pd: 98.2\%], worthlessness [HDI: $0.03,0.27$, pd: $97.2 \%$ ], and nervousness [HDI: $0.22,0.40$, pd: $100.0 \%$ ].

At the pd $>95 \%$ threshold, we observe only one reliable difference in distress mitigator performance, namely a difference among the earthquake-affected between satisfaction with the government for feelings of nervousness [HDI: 0.05, 0.28, pd: 98.7\%], see Figure 6BG3. Among the earthquake-affected, greater government satisfaction predicted a greater probability of feeling nervous "none of the time" and lower probability of feeling nervous "some of the time" when compared with responses among those whom the earthquake did not affect. However, we find no main effects of satisfaction with government for any of the other five Kessler-6 items besides nervousness, suggesting that satisfaction with the government was not generally a strong mitigator of distress in 2011. Therefore, we do infer a practically significant difference in this mitigator's performance among the earthquake-affected.

This comparative analysis of pandemic-lockdown distress with distress from a highly damaging natural disaster quantitatively clarifies distinctive COVID-19 lockdown features. Despite signals of mental health challenges during early COVID-19 lockdown, we estimate the magnitude of the mental health burden in New Zealand to have been greater following the Christchurch earthquakes. Additionally, earthquake-caused psychological distress was distributed across all six Kessler-6 items. Previous research identifies substantial personal losses and economic uncertainties as drivers of psychological distress following the Christchurch Earthquakes (23), with personality affecting magnitudes of change (24). The evidence we observe for amplified depression among those exposed to the earthquakes reflects harsh and sustained suffering following this natural disaster from damage to property, loss of life, and ongoing ground shaking

Data Archival. The data described in the paper are part of the New Zealand Attitudes and Values Study (NZAVS). Full copies of the NZAVS data files are held by all members of the NZAVS management team 
and advisory board. A de-identified dataset containing the variables analysed in this manuscript is available upon request from the corresponding author, or any member of the NZAVS advisory board for the purposes of replication or checking of any published study using NZAVS data. The code for all analyses and graphs is available on the Open Science Framework at: https://osf.io/bpved/.

ACKNOWLEDGMENTS. The New Zealand Attitudes and Values Study was approved by The University of Auckland Human Participants Ethics Committee on 03-June-2015 until 03-June-2018, and renewed on 05-September2017 until 03-June-2021. Reference Number: 014889. Our previous ethics approval statement for the 2009-2015 period is: The New Zealand Attitudes and Values Study was approved by The University of Auckland Human Participants Ethics Committee on 09-September-2009 until 09-September-2012, and renewed on 17-February-2012 until 09-September-2015. Reference Number: 6171. All participants granted informed written consent and The University of Auckland Human Participants Ethics Committee approved all procedures.

The New Zealand Attitudes and Values Study is supported by a grant from the Templeton Religion Trust (TRT0196). The funders had no role in the preparation of the article or the decision to publish.

\section{References.}

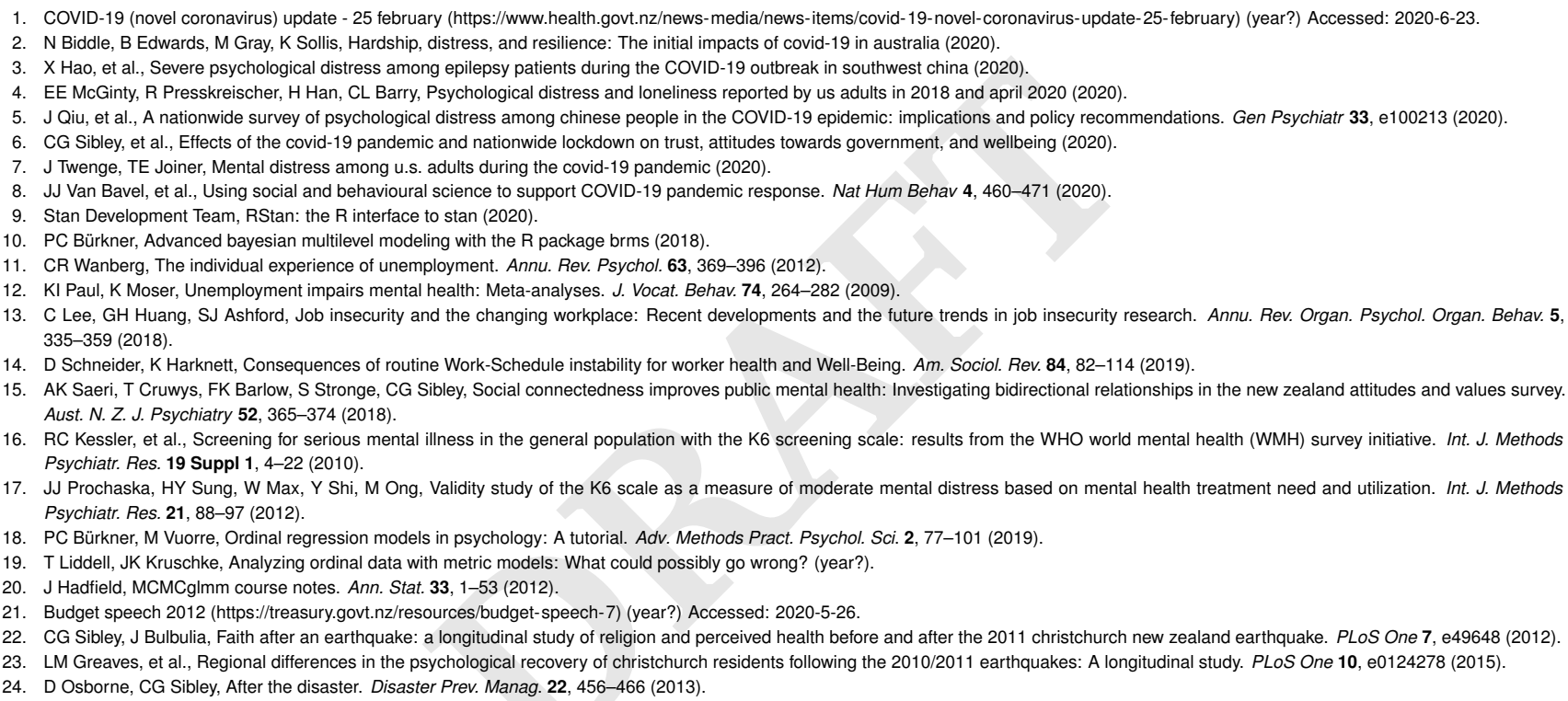

Supporting Information Appendix (SI). https://osf.io/ujbmf/ 


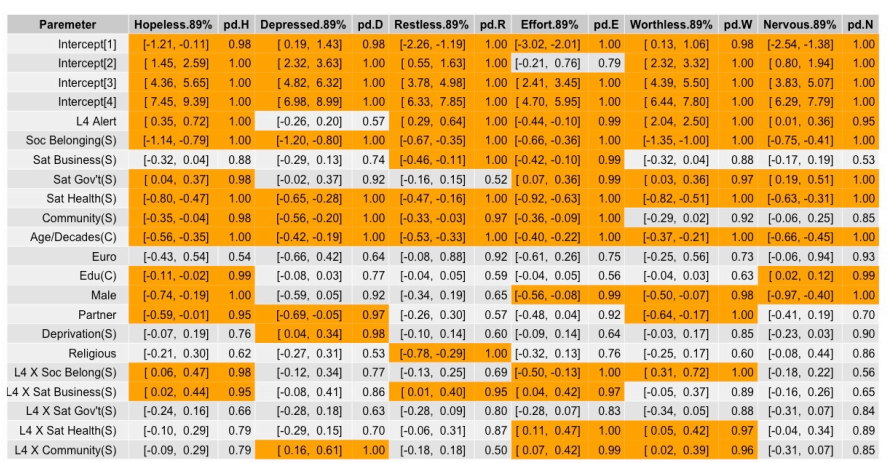

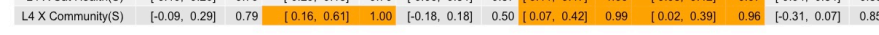

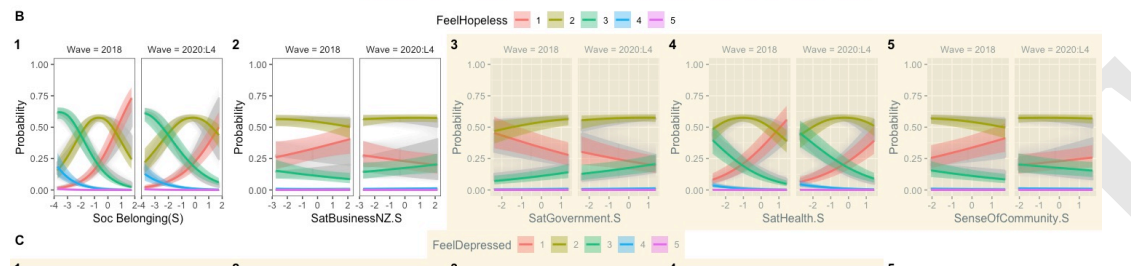
c

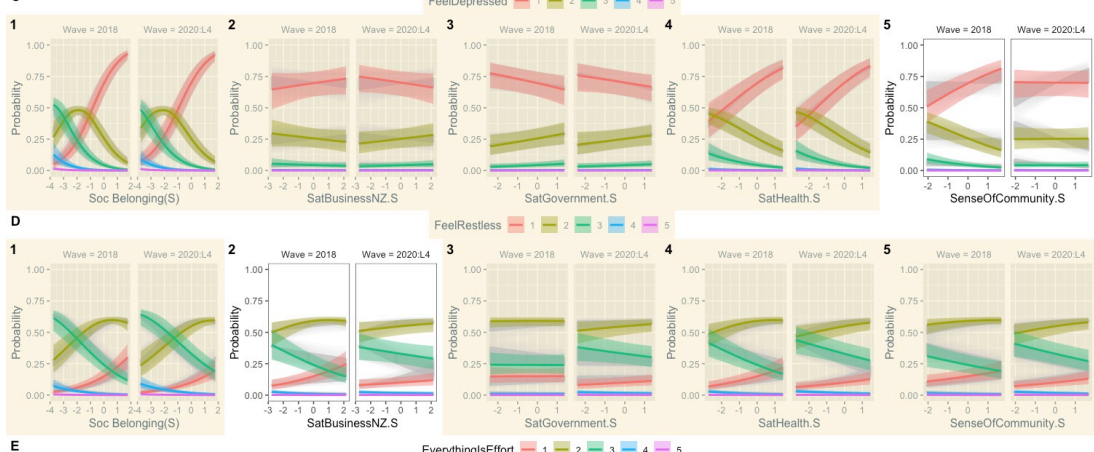
Everyth
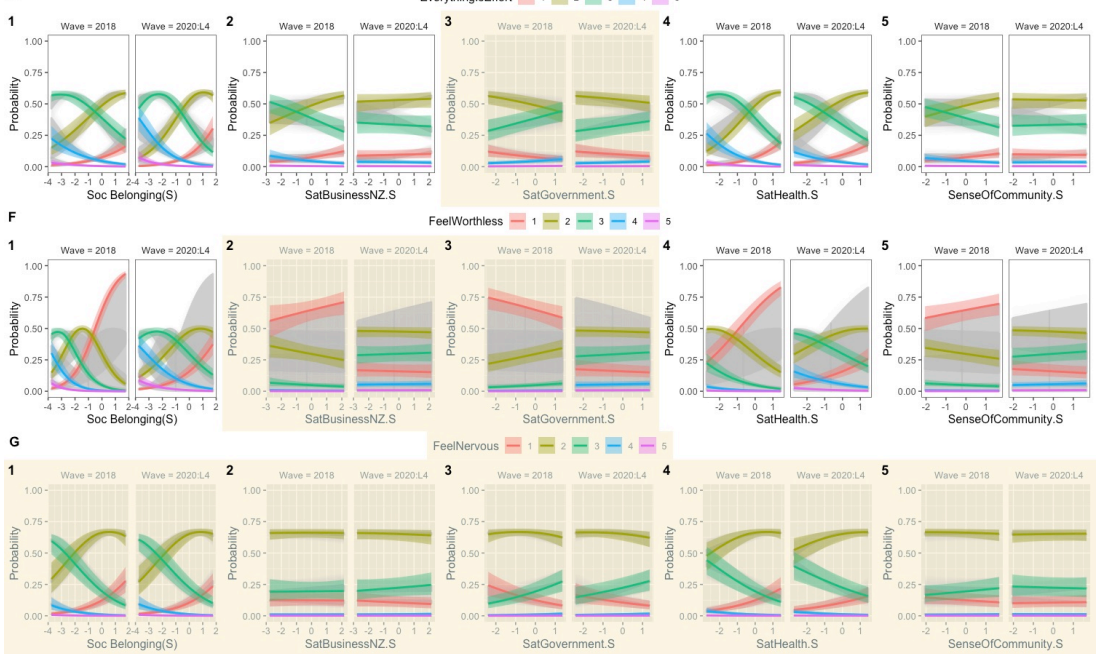

Fig. 2. (A) coefficient table for Kessler-6 psychological distress categories pre/during COVID-19 lockdown. Panels (B)-(F) show dimensions of distress; the five distress mitigators graphed in columns 1-5. White panels in the graph an orange cells int the table indicate reliable differences at the threshold of $\mathrm{pd}>95 \%$. During lockdown, health satisfaction and social belonging are the most important distress mitigator 

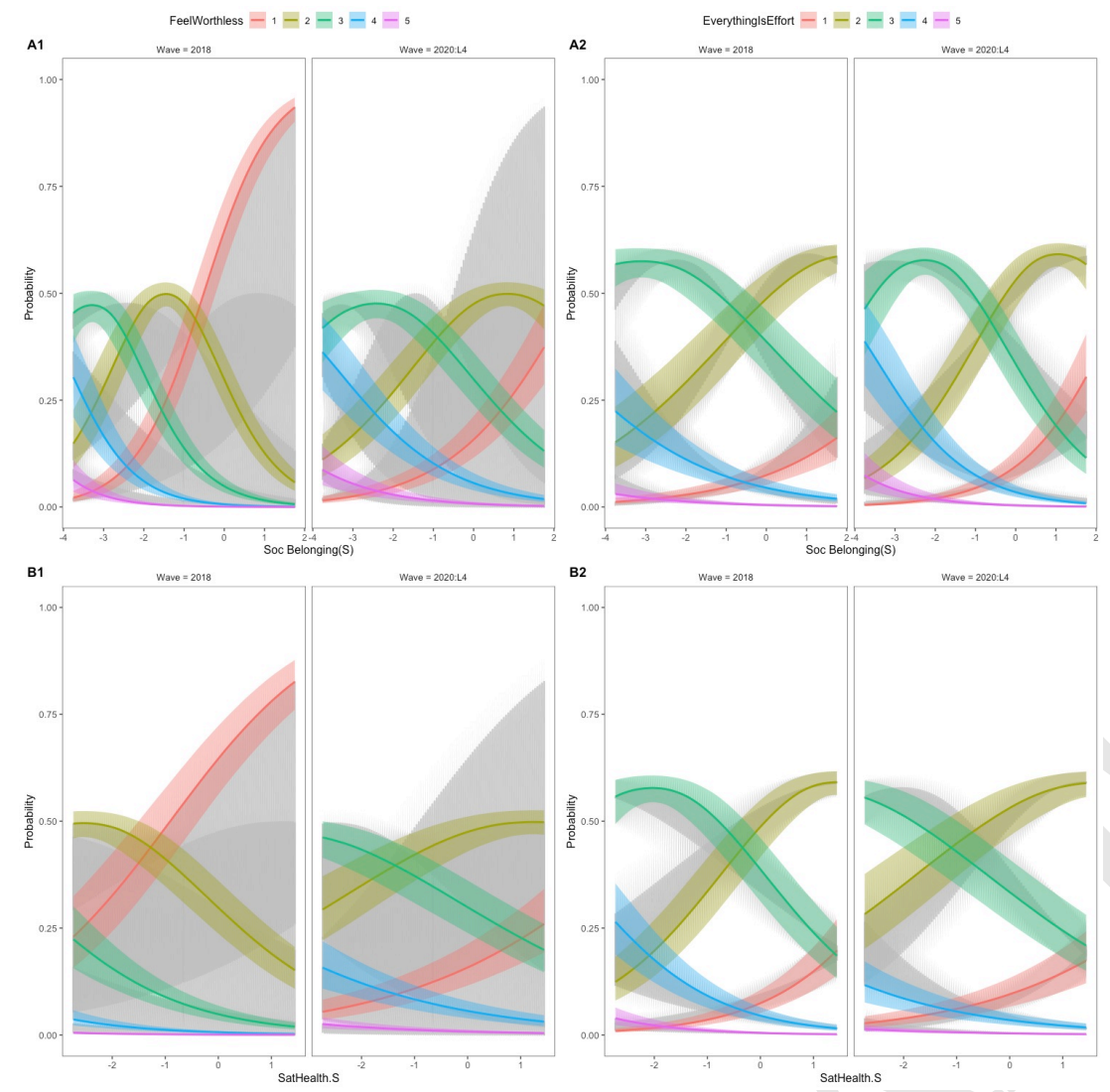

Fig. 3. (A1) During early lockdown, there was a strong attenuation of social belonging on feelings of worthlessness (response 1: "none of the time" and response 2: "a little of the time"), though with comparably worse outcomes for those who are low on social belonging (elevation in response 3: "some of the time", response 4: "most of the time", and response 5: "all of the time"). (A2) During early lockdown, social belonging functioned as a pathway mechanism to facilitate relief from feelings of effort (responses 1,2$)$. Moreover, low social belonging was associated with a greater perception of effort (responses 3-5). (B1) During early lockdown, there was a strong attenuation of health satisfaction on feelings of worthlessness across all levels of response. (B2) Health satisfaction was not a pathway effect for a reduction in feelings that everything is an effort. Rather, change occurred at the low end of health satisfaction. During the lockdown, those low in health satisfaction brought a sense of relief.

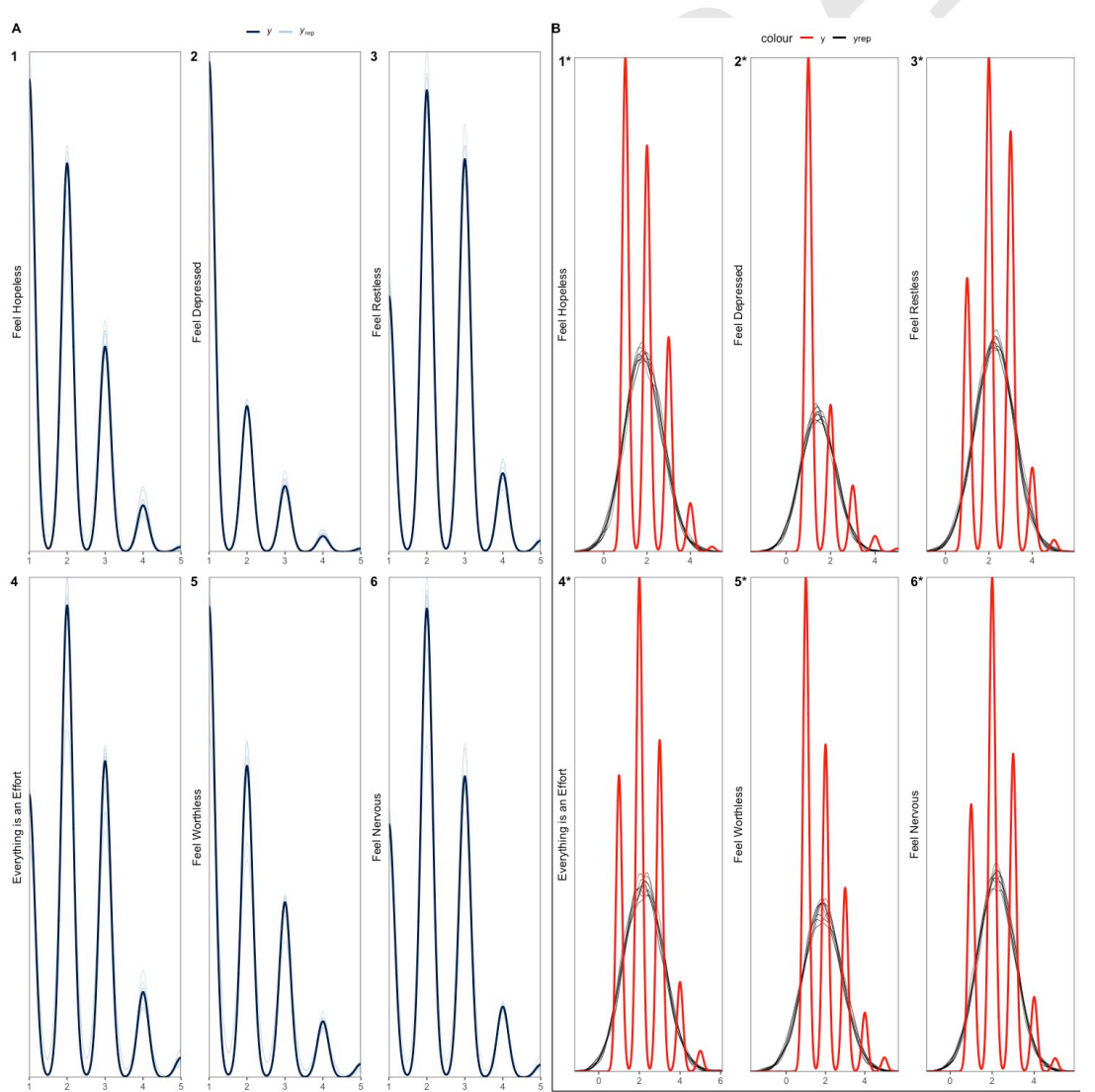

Fig. 4. (A)PP checks show good fit for cumulative categorical model of distress dimensions pre/during COVID19 lockdown. (B) PP checks reveal how poor the model fits responses under the assumption of a Gaussian distribution. We advise psychological scientists to avoid the Gaussian distribution when inferring Kessler-6 outcomes. 


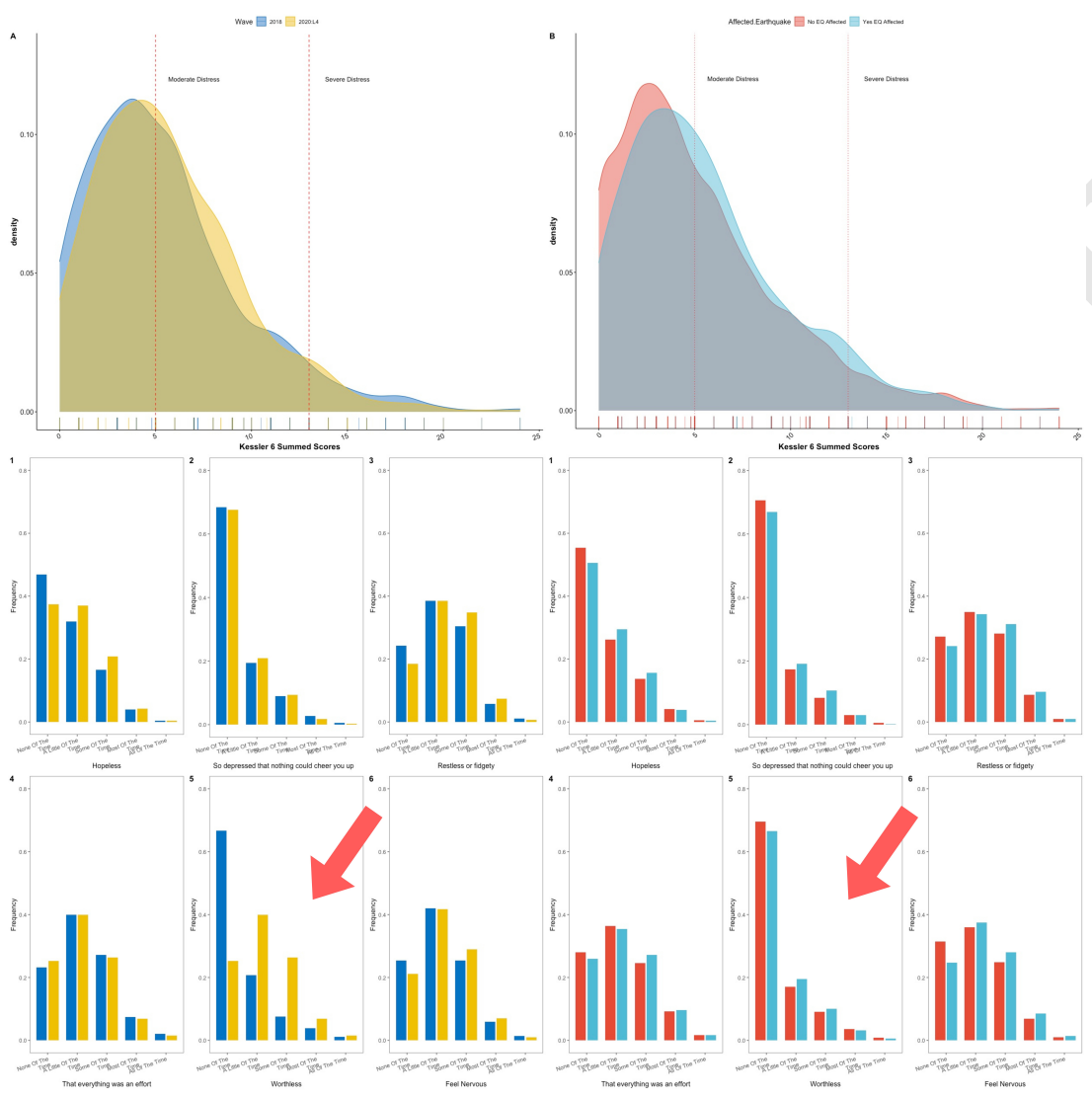

Fig. 5. Comparison of Kessler-6 distress scores (summed and individual items) in COVID-19 lockdown and Christchurch samples reveals an anomalous surge in feelings of worthlessness during COVID-19 lockdown. Greater challenges are evident following Christchurch earthquakes in feelings of depression (B2) and feelings nervousness (B6) 


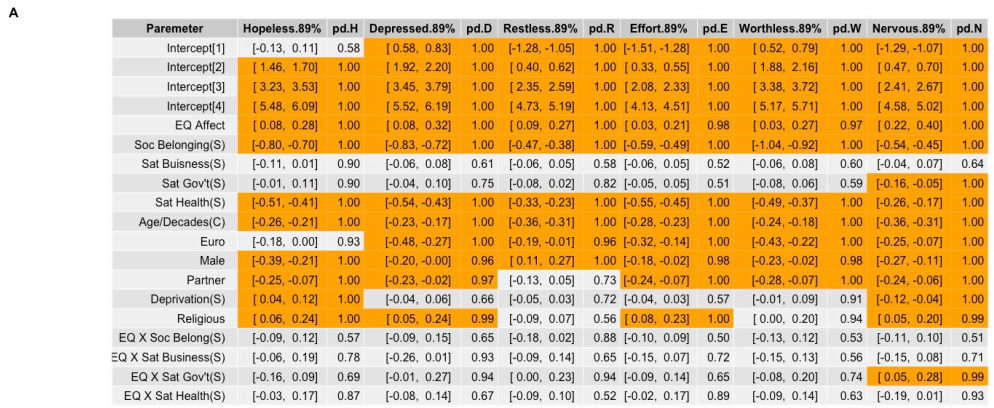

B

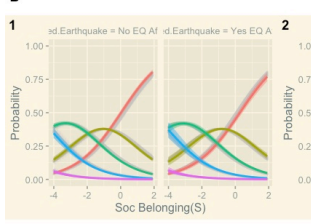

c
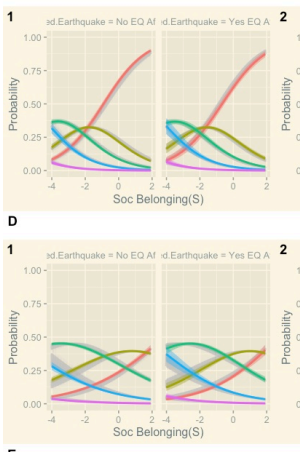

E
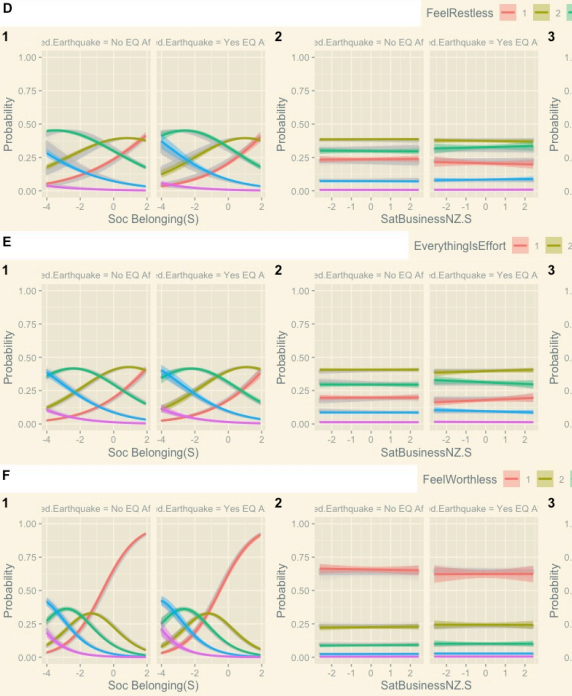

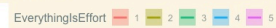
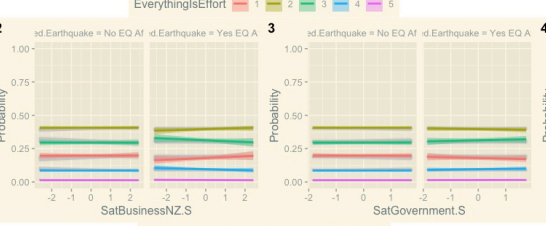

Feel Worthess $-1, A_{2}=3+4=$
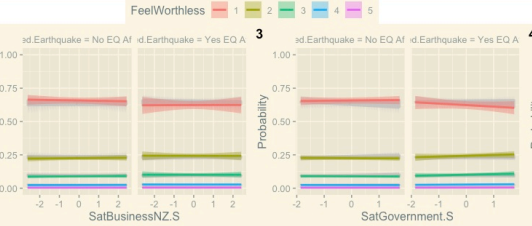

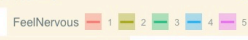

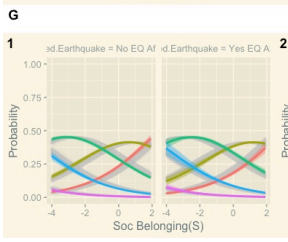

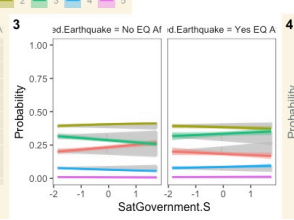
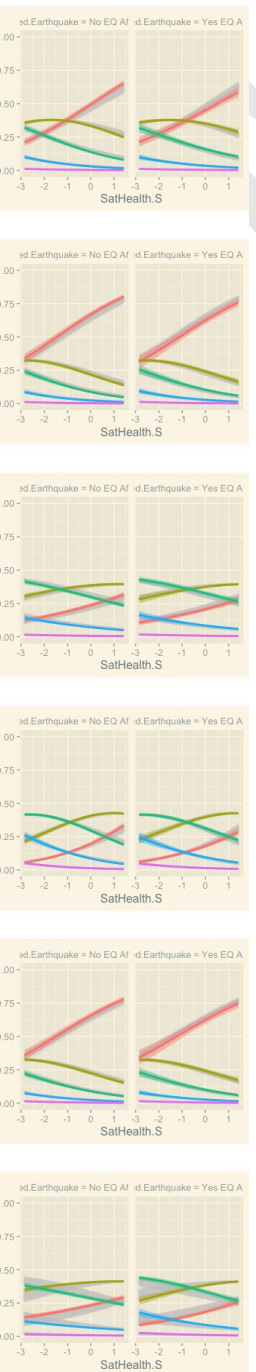

Fig. 6. Christchurch Earthquake Analysis (A) coefficient table for estimates for Kessler-6 psychological distress categories among those affected/not affected by the 2011 Christchurch earthquakes (year sampled 2011). Graphs show differences in the relationship of four psychological distress mitigation indicators (columns 1-4) for the six indicators of psychological distress (rows (B-F). We observe increases in all areas of mental distress including feelings of depression and feelings that everything is an effort. We detect a flattening effect in which satisfaction with the government fails to predict nervousness among those who were affected by the Christchurch earthquakes. The relationships between the other distress-mitigators and distress indicators were similar among those affected by the Christchurch earthquakes and those who were not affected. 